\title{
Universal Pseudo-Differential Filter Using DDCC and DVCCs
}

\author{
Ondrej Sladok, Jaroslav Koton, Norbert Herencsar \\ Department of Telecommunications, Brno University of Technology, \\ Technicka 3082/12, 61600 Brno, Czech Republic \\ sladok@phd.feec.vutbr.cz
}

\begin{abstract}
In the paper, a universal preudo-differential second-order filter operating in voltage mode, where both intup and output are differential, is presented. The circuit is formed by one differential difference current conveyor (DDCC), two differential voltage current conveyors (DVCCs), and five passive elements. The filter is characterized by high input impedance, minimum number of passive elements that are all grounded, and high common-mode rejection ratio (CMRR). The proposed filter structure is able to realize all five standard frequency filter responses. Non-ideal analysis has been performed by considering the real parasitic parameters of the active elements. The optimization of passive element values has been done in terms of minimal shift of the pole-frequency and to obtain the maximum stop-band attenuation of the high-pass filter response. Functionality is verified by simulations and experimental measurements using readily available integrated circuit UCC-N1B 0520.
\end{abstract}

Index Terms-Pseudo-differential filter; voltage-mode; current conveyor; CMRR.

\section{INTRODUCTION}

Frequency filters are widely used in the vast majority of electrical equipments such as in video signal processing, communication systems, telephone circuitry, broadcasting systems, control and instrumentation systems, etc. Therefore, a significant number of filters are available in the open literature using various types of function blocks. For designing frequency filters (fully differential, single-ended, or pseudo-differential) operational transconductance amplifiers (OTAs) [1]-[4] current follower transconductance amplifiers (CFTAs) [5], [6], current differencing transconductance amplifiers (CDTAs) [7], [8] were commonly used during the last two decades. Frequency filters can also be utilized using basic types of current conveyors (CC) [9]-[11], fully differential current conveyors (FDCC) [12], [13], differential voltage current conveyors (DVCC) [14], [15], or differential difference current conveyor (DDCC) [16], [17]. Currently, the design of function blocks is emphasized on low supply voltage and low-power consumption solutions [18]-[21]. However, by

Manuscript received 28 December, 2016; accepted 18 June, 2017.

Research described in this paper was financed by the National Sustainability Program under grant LO1401 and by the Czech Science Foundation under grant no. 16-11460Y. For the research, infrastructure of the SIX Center was used. decreasing the supply voltage of function blocks it has the consequence in reduced dynamic range of the signals being processed due to reduced signal-to-noise ratio (SNR). Therefore, an increased interest in designing fullydifferential frequency filters using various types of new active elements can be observed [22]-[28]. In comparison to their single-ended frequency filter counterparts, differential filters are generally capable to maintain sufficient SNR, are characterized by lower total harmonic distortion (THD), feature high common-mode signal rejection (CMRR), and furthermore with reduced input noise [29]. To design a fullydifferential frequency filter, mostly a single-ended prototype is mirrored around the ground plane, which requires the usage of fully-differential active elements and also symmetry in passive elements can be observed [30]. This design technique results in quite complex structures and therefore the pseudo-differential filters were presented in which both input and output are assumed in differential form, but the internal structure of the filter is single-ended [16], [17], [31]-[32].

In this paper, a new second-order voltage-mode universal pseudo-differential filter using three active elements (one DDCC and two DVCCs) is presented. Together with the active elements only five passive elements (two capacitors and three resistors), each of them grounded, are used. Theoretically, the input-impedance of the proposed filter is infinitely high and hence it is suitable for easy cascading. Even if it is pseudo-differential, such filter still exhibits with high CMRR and low signal distortion. The proposed universal pseudo-differential filter realizes all five standard types of frequency filters, i.e. (low-pass, high-pass, bandpass, band-reject, and all-pass). The behavior of the filter was verified by simulations and furthermore by experimental measurements that show the functionality of the proposed solution.

\section{DESCRIPTION OF DVCC AND DDCC}

For the design of universal pseudo-differential filter the DVCC and DDCC active elements have been used, which schematic symbols are shown in Fig. 1. First, the DVCC is a five terminal building block with two high-impedance voltage inputs $Y_{1}$ and $Y_{2}$, one low-impedance current input $X$, and two high-impedance current outputs $Z_{1+}$ and $Z_{1}$.. Relation between the terminal currents and voltages is 
described by:

$$
\left\{\begin{array}{l}
V_{\mathrm{x}}=V_{\mathrm{y} 1}-V_{\mathrm{y} 2}, \\
I_{\mathrm{y} 1}=I_{\mathrm{y} 2}=0 \\
I_{\mathrm{z} 1+}=I_{\mathrm{x}}, \\
I_{\mathrm{z} 1-}=-I_{\mathrm{x}} .
\end{array}\right.
$$

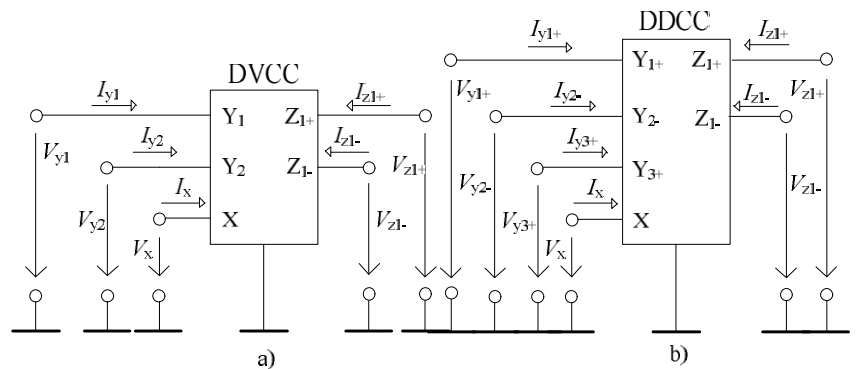

Fig. 1. Schematic symbols: a) DVCC b) DDCC.

The DDCC is a six-terminal building block with three high-impedance voltage inputs $\mathrm{Y}_{1+}, \mathrm{Y}_{2-}$ and $\mathrm{Y}_{3+}$, a lowimpedance current input $\mathrm{X}$, and two high-impedance current outputs $\mathrm{Z}_{1+}$ and $\mathrm{Z}_{1 \text {.. }}$ The relation between terminal currents and voltages is given as:

$$
\left\{\begin{array}{l}
V_{\mathrm{x}}=V_{\mathrm{y} 1+}-V_{\mathrm{y} 2-}+V_{\mathrm{y} 3+} \\
I_{\mathrm{y} 1+}=I_{\mathrm{y} 2-}=I_{\mathrm{y} 3+}=0 \\
I_{\mathrm{z} 1+}=I_{\mathrm{x}}, I_{\mathrm{z} 1-}=-I_{\mathrm{x}} .
\end{array}\right.
$$

\section{PSEUdo-DiffERENTIAL FILTERS}

Generally, when analyzing the differential circuits operating in voltage mode, the following relations are assumed [29]:

$$
\left\{\begin{array}{l}
v_{1 \mathrm{~d}}=v_{1+}-v_{1-}, \\
v_{2 \mathrm{~d}}=v_{2+}-v_{2-}, \\
v_{1 \mathrm{c}}=\frac{v_{1+}-v_{1-}}{2},
\end{array}\right.
$$

where $v_{1 \mathrm{~d}}, v_{2 \mathrm{~d}}$ and $v_{1 \mathrm{c}}$ denote differential input voltage, the differential output voltage, and the common-mode input voltage, respectively. Signal $v_{1 \mathrm{~d}}$ is the difference between the two input signals $v_{1+}$ and $v_{1-}$, while $v_{1 \mathrm{c}}$ is expressing common-mode input signal as the average of the two input

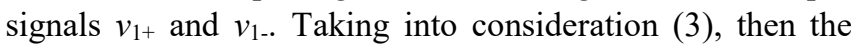
differential output voltage $v_{2 \mathrm{~d}}$ is defined as follows

$$
v_{2 \mathrm{~d}}=v_{1 \mathrm{~d}} \times A_{\mathrm{dm}}+v_{1 \mathrm{c}} \times A_{\mathrm{cm}},
$$

where $A_{\mathrm{dm}}$ and $A_{\mathrm{cm}}$ are differential and common-mode signal gains, respectively. The rejection of common-mode signal is expressed using CMRR as [29]

$$
C M R R=20 \log \left(\frac{A_{\mathrm{dm}}}{A_{\mathrm{cm}}}\right),
$$

which determines the ability of an active element or a differential structure to suppress unwanted common-mode input signal that is common to both inputs, to the desired differential input signal. In ideal case $A_{\mathrm{cm}}=0$ and hence CMRR is infinite.

As mentioned in the Section I., the fully-differential filters are designed using e.g. the mirroring technique, [30]. However, it is obvious from the mathematical description point of view (3)-(5) that for sake of analysis of such function blocks only the input and output signals are considered. Therefore, it is possible to describe and propose so-called pseudo-differential structures, which have differential input and output voltage terminals, however, the inner circuit topology is non-differential. These types of filters still provide high CMRR similarly as fully-differential structures and furthermore, are less complex because of the internal non-differential structure. In practice, in combination with fully-differential circuits the pseudodifferential function blocks can be used as the last section(s) of front-end analog signal processing path, where very high CMRR is no more required [31].

\section{Proposed Universal PseUdo-Differential Filter}

For the design of a pseudo-differential filter, similarly to the design of fully-differential filters, a single ended prototype is also used. However, as described in [31], in case of pseudo-differential filters only the non-differential input and output are to be transformed into differential one.

\section{A. Single-Ended Prototype}

The single-ended structure shown in Fig. 2 was described in [34] and it is a pattern for the proposed universal pseudodifferential filter. The prototype is realized using three active elements, i.e. two DVCCs, one second generation current conveyor (CCII-3) and four passive elements such as two grounded capacitors and two grounded resistors. This filter uses known connection Y-Z of the conveyors, which advantage is applying the input signal to high-impedance terminal $\mathrm{Y}$ of the active element and it ensures its simple connection in cascade.

Circuit given in Fig. 2 allows realizing four basic singleended second-order frequency filter responses (LP, BP, HP, and $\mathrm{BR})$, which are described by the following equations:

$$
\left\{\begin{array}{l}
v_{\mathrm{LP}}=\frac{1}{s^{2} C_{1} C_{2} R_{1} R_{2}+s C_{2} R_{2}+1}, \\
v_{\mathrm{BP}}=\frac{s C_{2} R_{2}}{s^{2} C_{1} C_{2} R_{1} R_{2}+s C_{2} R_{2}+1}, \\
v_{\mathrm{HP}}=\frac{s^{2} C_{1} C_{2} R_{1} R_{2}}{s^{2} C_{1} C_{2} R_{1} R_{2}+s C_{2} R_{2}+1}, \\
v_{\mathrm{BR}}=\frac{s^{2} C_{1} C_{2} R_{1} R_{2}+1}{s^{2} C_{1} C_{2} R_{1} R_{2}+s C_{2} R_{2}+1} .
\end{array}\right.
$$

A detailed analysis of this filter and its performance verification can be found in [34].

\section{B. Proposed Universal Pseudo-Differential Filter}

Using the techniques described in [31], the proposed second-order pseudo-differential frequency filter is shown in Fig. 3. 


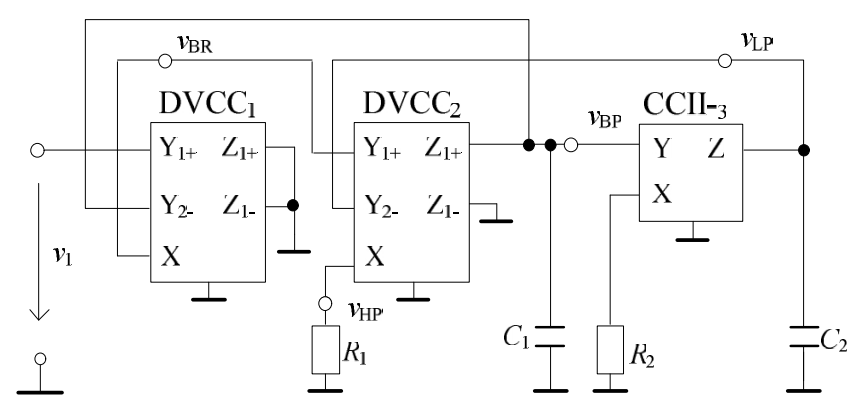

Fig. 2. Single-ended filter as prototype for the pseudo-differential filter design.

The proposed filter is composed from one DDCC and two DVCCs. The structure also includes four passive elements, namely three resistors and two capacitors. To obtain a differential input, the $\mathrm{DVCC}_{1}$ in Fig. 2 was substituted by $\mathrm{DDCC}_{1}$ as it can be observed in Fig. 3. Hence, the differential input signal is applied to the input terminals $\mathrm{Y}_{1+}$ and $\mathrm{Y}_{2-}$ of the active element. In order to obtain a bandreject response, the $\mathrm{DVCC}_{2}$ in Fig. 2 has been extended via $\mathrm{Z}_{2}$ - output terminal, whereas the resistor $R_{3}$ has been added with the same value as resistor $R_{1}$. To obtain differential band-pass response, the $\mathrm{CCII}_{-3}$ in Fig. 2 was substituted by $\mathrm{DVCC}_{3}$, where the inner voltage inverter is used. The remaining passive elements $R_{1}, R_{2}, C_{1}$, and $C_{2}$ are unchanged.

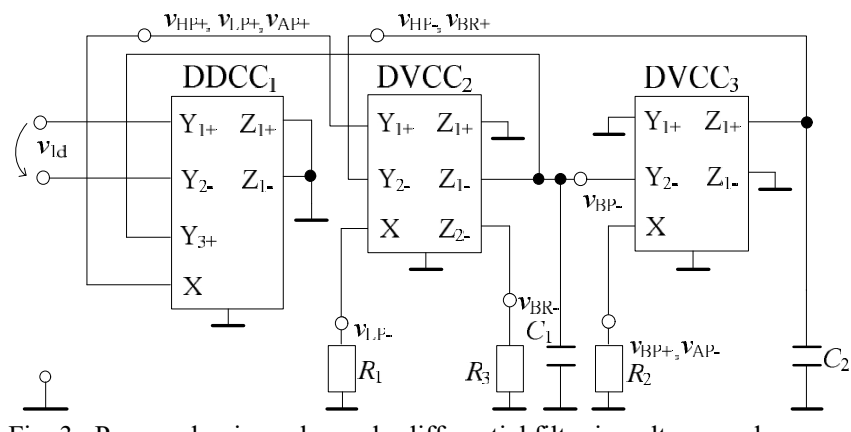

Fig. 3. Proposed universal pseudo-differential filter in voltage mode.

The advantageous features of the pseudo-differential filter are the same as they are in case of the single-ended prototype. Furthermore, it features with high CMRR, it is characterized by lower THD, and its structure is generally less complex in comparison with a fully-differential solution designed by the mirroring technique.

\section{1) Ideal Pseudo-Differential Filter Analysis}

Assuming ideal active elements differential-output voltage for individual filters, i.e. high-pass, low-pass, all-pass, bandpass, band-reject transfer functions can be determined as described below. For an instance, using the notation (3)-(5), the positive and negative output voltage of the differential high-pass filter can be given as:

$$
\begin{aligned}
& v_{\mathrm{HP}+}=\frac{s^{2} C_{1} C_{2} R_{1} R_{2}+1}{s^{2} C_{1} C_{2} R_{1} R_{2}+s C_{2} R_{2}+1} \times v_{1 \mathrm{~d}}+0 \times v_{1 \mathrm{c}}, \\
& v_{\mathrm{HP}-}=\frac{1}{s^{2} C_{1} C_{2} R_{1} R_{2}+s C_{2} R_{2}+1} \times v_{1 \mathrm{~d}}+0 \times v_{1 \mathrm{c}},
\end{aligned}
$$

whereas for differential-output voltage $v_{\mathrm{HPd}}$ it applies:

$$
v_{\mathrm{HPd}}=\frac{s^{2} C_{1} C_{2} R_{1} R_{2}}{s^{2} C_{1} C_{2} R_{1} R_{2}+s C_{2} R_{2}+1} \times v_{1 \mathrm{~d}}+0 \times v_{1 \mathrm{c}} .
$$

Comparing (9) with (4) we get differential gain

$$
A_{\mathrm{dmHP}}=\frac{s^{2} C_{1} C_{2} R_{1} R_{2}}{s^{2} C_{1} C_{2} R_{1} R_{2}+s C_{2} R_{2}+1},
$$

whereas the common-mode signal gain is zero. Remaining four frequency filter responses can be obtained using the corresponding positive and negative output as defined in Fig. 3. Hence, low-pass differential gain is the following

$$
A_{\mathrm{dmLP}}=\frac{1}{s^{2} C_{1} C_{2} R_{1} R_{2}+s C_{2} R_{2}+1},
$$

all-pass differential gain can be expressed as

$$
A_{\mathrm{dmAP}}=\frac{s^{2} C_{1} C_{2} R_{1} R_{2}-s C_{2} R_{2}+1}{s^{2} C_{1} C_{2} R_{1} R_{2}+s C_{2} R_{2}+1}
$$

band-pass differential gain can be calculated as

$$
A_{\mathrm{dmBP}}=\frac{2 \times s C_{2} R_{2}}{s^{2} C_{1} C_{2} R_{1} R_{2}+s C_{2} R_{2}+1},
$$

and for $R_{1}=R_{3}$ band-reject differential gain can be found as

$$
A_{\mathrm{dmBR}}=\frac{s^{2} C_{1} C_{2} R_{1} R_{2}+1}{s^{2} C_{1} C_{2} R_{1} R_{2}+s C_{2} R_{2}+1} .
$$

Due to the fact that the common-mode gain $A_{\mathrm{cm}}$ for all frequency filters equals to 0 , the CMRR equals to infinite, if ideal active elements are considered. For universal pseudodifferential filter the characteristic pole-frequency $\omega_{0}$ and quality factor $Q$ are defined as:

$$
\begin{gathered}
\omega_{0}=\frac{1}{\sqrt{C_{1} C_{2} R_{1} R_{2}}}, \\
Q=\sqrt{\frac{C_{1} R_{1}}{C_{2} R_{2}}} .
\end{gathered}
$$

\section{2) Non-Ideal Analysis of Pseudo-Differential Filter}

Taking into consideration the non-idealities of the active elements [31], [34] the terminal relations of DVCC can be expressed as:

$$
\left\{\begin{array}{l}
V_{\mathrm{x}}=\beta_{1} V_{\mathrm{y} 1}-\beta_{2} V_{\mathrm{y} 2}, \\
I_{\mathrm{z} 1+}=\alpha_{1} I_{\mathrm{x}}, \\
I_{\mathrm{z} 1-}=-\alpha_{2} I_{\mathrm{x}},
\end{array}\right.
$$

where $\beta_{\mathrm{j}}=1-\varepsilon_{\mathrm{vj}}$ and $\alpha_{\mathrm{k}}=1-\varepsilon_{\mathrm{ik}}$ (for $\mathrm{j}=\{1,2\}$ and $\mathrm{k}=\{1,2\})$ are the voltage and current gains of the DVCC, and $\left|\varepsilon_{\mathrm{vj}}\right| \ll 1$ a $\left|\varepsilon_{\mathrm{ik}}\right| \ll 1$ denote the voltage and current 
tracking errors, respectively.

Similarly, the non-ideal behavior of the DDCC can be defined as follows:

$$
\left\{\begin{array}{l}
V_{\mathrm{x}}=\delta_{1} V_{\mathrm{y} 1+}-\delta_{2} V_{\mathrm{y} 2-}+\delta_{3} V_{\mathrm{y} 3+} \\
I_{\mathrm{z} 1+}=\gamma_{1} I_{\mathrm{x}} \\
I_{\mathrm{z} 1-}=-\gamma_{2} I_{\mathrm{x}}
\end{array}\right.
$$

where $\delta_{\mathrm{m}}=1-\varepsilon_{\mathrm{vm}}$ and $\gamma_{\mathrm{n}}=1-\varepsilon_{\mathrm{in}}$ (for $\mathrm{m}=\{1,2,3\}$ and $\mathrm{n}=\{1,2\})$ are the voltage and current gains of the DDCC, and $\left|\varepsilon_{\mathrm{vm}}\right| \ll 1$ and $\left|\varepsilon_{\text {in }}\right| \ll 1$ denote the voltage and current tracking errors.

To investigate the influence of the non-ideal voltage and current gains (17) and (18) of the active elements, the highpass response was chosen for further analysis. The differential and common-mode signal can be determined as:

$$
\left\{\begin{array}{l}
A_{\mathrm{dmHP}}=\frac{\left(\delta_{1}+\delta_{2}\right)}{2} \times \\
\times \frac{s^{2} C_{1} C_{2} R_{1} R_{2}+\beta_{2} \alpha_{1} \gamma_{2}\left(\delta_{2}-\delta_{1}\right)}{s^{2} C_{1} C_{2} R_{1} R_{2}+s C_{2} R_{2} \delta_{1} \delta_{3} \gamma 2+\delta_{2} \gamma_{2} \beta_{2} \alpha_{1}} \\
A_{\mathrm{cmHP}}=\left(\delta_{1}-\delta_{2}\right) \times \\
\times \frac{s^{2} C_{1} C_{2} R_{1} R_{2}+\beta_{2} \alpha_{1} \gamma_{2}\left(\delta_{2}-\delta_{1}\right)}{s^{2} C_{1} C_{2} R_{1} R_{2}+s C_{2} R_{2} \delta_{1} \delta_{3} \gamma_{2}+\delta_{2} \gamma_{2} \beta_{2} \alpha_{1}}
\end{array}\right.
$$

and according to (5), the CMRR equals to

$$
C M R R=20 \log \left|\frac{\left(\delta_{1}+\delta_{2}\right)}{2\left(\delta_{1}-\delta_{2}\right)}\right| .
$$

From (15) it is obvious that high rejection of the commonmode signal is ensured in case when $\delta_{1} \approx \delta_{2}$.

Due to non-ideal voltage and current gains of active elements, the characteristic pole-frequency and quality factor modify to:

$$
\left\{\begin{array}{l}
\omega_{0}=\sqrt{\frac{\delta_{2} \gamma_{2} \beta_{2} \alpha_{1}}{C_{1} C_{2} R_{1} R_{2}}}, \\
Q=\frac{1}{\delta_{1} \delta_{3}} \sqrt{\frac{\delta_{2} \beta_{2} \alpha_{1}}{\gamma_{2}}} \sqrt{\frac{C_{1} R_{1}}{C_{2} R_{2}}} .
\end{array}\right.
$$

As voltage and current tracking errors of active elements are minimal [35], effect of non-ideal voltage and current gains on filter properties is not significant.

The performance of the filter is more affected by the parasitic impedances of the active elements. In Fig. 4, the most significant [31], [34] parasitics are represented by $R_{\mathrm{v}}$, $R_{\mathrm{w}}, C_{\mathrm{v}}, C_{\mathrm{w}}$, that describe the finite impedance of the $\mathrm{Y}$ and $\mathrm{Z}$ terminals of the active elements.

Re-analysis of the filter yields the following differential gain of the high-pass filter

$$
\begin{aligned}
& A_{\mathrm{dmHP}}= \\
& s^{2} C_{1}^{\prime} C_{2}^{\prime} R_{1}^{\prime} R_{2}^{\prime} R_{\mathrm{v}} R_{\mathrm{w}}+s R_{1}^{\prime} R_{2}^{\prime}\left(C_{2}^{\prime} R_{\mathrm{v}}+C_{1}^{\prime} R_{\mathrm{w}}\right)+ \\
& =\frac{+R_{1}^{\prime} R_{2}^{\prime}}{s^{2} C_{1}^{\prime} C_{2}^{\prime} R_{1}^{\prime} R_{2}^{\prime} R_{\mathrm{v}} R_{\mathrm{w}}+s R_{2}^{\prime}\left(C_{2}^{\prime} R_{\mathrm{v}} R_{\mathrm{w}}+C_{1}^{\prime} R_{1} R_{\mathrm{w}}+\right.} \\
& \left.+C_{2}^{\prime} R_{1} R_{\mathrm{v}}\right)+R_{1}^{\prime} R_{2}^{\prime}+R_{2}^{\prime} R_{\mathrm{W}}+R_{\mathrm{v}} R_{\mathrm{w}}
\end{aligned}
$$

where $\quad C_{1}^{\prime}=C_{1}+C_{\mathrm{w}}, \quad C_{2}^{\prime}=C_{2}+C_{\mathrm{v}}, \quad R_{1}^{\prime}=R_{1}+R_{\mathrm{x}}$, $R_{2}^{\prime}=R_{2}+R_{\mathrm{x}}, \quad R_{\mathrm{v}}=R_{\mathrm{z} 1}\left\|R_{\mathrm{y} 2}, \quad R_{\mathrm{w}}=R_{\mathrm{z} 2}\right\| R_{\mathrm{y} 2} \| R_{\mathrm{y} 3}$, $C_{\mathrm{v}}=C_{\mathrm{z} 1}+C_{\mathrm{y} 2}, C_{\mathrm{w}}=C_{\mathrm{z} 2}+C_{\mathrm{y} 2}+C_{\mathrm{y} 3}$, if $R_{\mathrm{y}}, R_{\mathrm{x}}, R_{\mathrm{z}}$ and $C_{\mathrm{y}}, C_{\mathrm{y}}$ are parasitic resistors and capacitors, respectively, wheras for sake of simplicity, the voltage and current gains of the active elements were assumed to be unity.

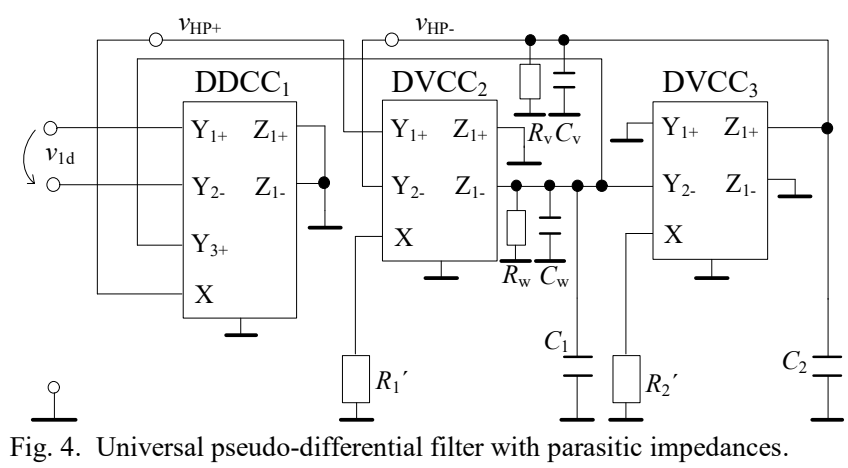

For proper function of frequency filter it has to apply $R_{1}$; $R_{2} \ll R_{\mathrm{v}} ; R_{\mathrm{w}}$, and the following pole-frequency and quality factor can be given:

$$
\begin{gathered}
\omega_{0} \approx \frac{1}{\sqrt{C_{1}^{\prime} C_{2}^{\prime} R_{1}^{\prime} R_{2}^{\prime}}}, \\
Q \approx \sqrt{\frac{C_{1}^{\prime} R_{1}^{\prime}}{C_{2}^{\prime} R_{2}^{\prime}}} .
\end{gathered}
$$

To suppress the parasitic behavior of the active elements as much as possible, the values of passive elements should be kept as: $C_{1} \gg C_{\mathrm{v}}, C_{2}$ » $C_{\mathrm{w}}$ and $R_{1} ; R_{2} \gg R_{\mathrm{x}}$

\section{Simulation AND EXPERIMENTAL MEASUREMENTS}

The performance of the proposed pseudo-differential filter has been evaluated by simulations PSpice and later also by experimental measurements. To realize the active elements, the universal current conveyor UCC-N1B 0520 [35] was used. The parameters of non-ideal properties of active elements are taken from the datasheet [35]. Simulations assume parasitic impedances of these active elements, trying to acquire the most optimal values of passive elements.

\section{A. Optimization of Universal Pseudo-Differential Filter}

The optimization of pseudo-differential filter has been done in two phases, whereas the high-pass response was assumed. The aim of this optimization process is to obtain the most optimal values of passive elements. During the 
optimization, theoretical pole-frequency $f_{0}=100 \mathrm{kHz}$ and quality factor $Q=0.707$ were assumed.

The first optimization phase was to minimize the shift of the pole-frequency $f_{0 \text { sim }}$ determined by (23) from theoretical frequency $f_{0 \text { teo }}$ defined by (15). The shift between these frequencies is evaluated as their ratio, whereas the optimal value of such ratio is unity. In Fig. 5 the ratio between theoretical and simulated pole-frequency is shown.

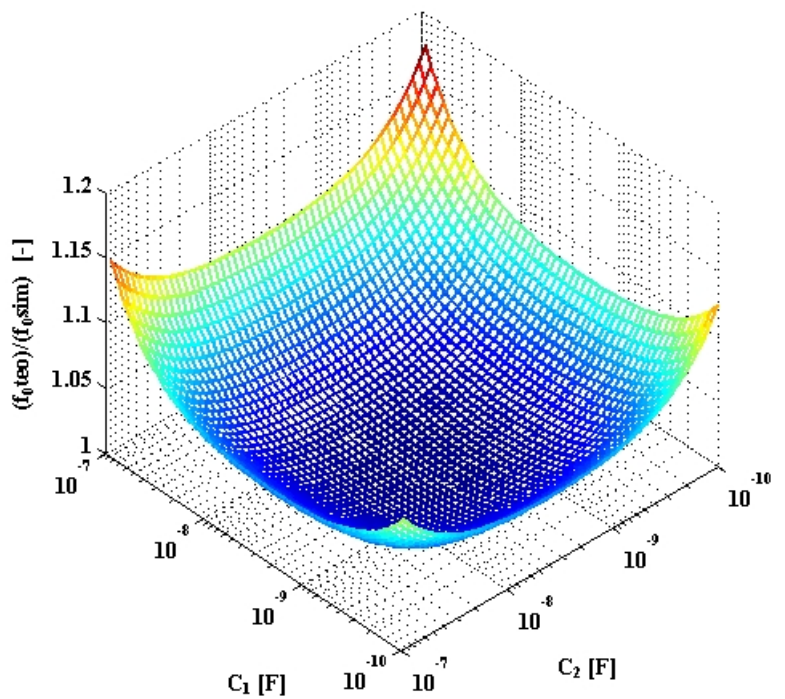

Fig. 5. Variant of theoretical pole-frequency in proportion of simulated frequency for $Q=0.707$.

The second optimization phase focused on obtaining the highest attenuation in the stop-band of the high-pass frequency response, i.e. to minimize the ratio of the $s^{0}$ terms in the numerator and denominator of (22)

$$
A_{\mathrm{dmHP}-\mathrm{SB}}=\frac{R_{1}^{\prime} R_{2}^{\prime}}{R_{1}^{\prime} R_{2}^{\prime}+R_{2}^{\prime} R_{\mathrm{w}}+R_{\mathrm{v}} R_{\mathrm{w}}} .
$$

Varying the values of capacitors $C_{1}$ and $C_{2}$, the ratio $A_{\text {dmHP_SB }}$ is shown in Fig. 6 , whereas the values of resistors $R_{1}$ and $R_{2}$ were determined using (15) and (16) for theoretical pole-frequency $f_{0}=100 \mathrm{kHz}$ and quality factor $Q=0.707$.

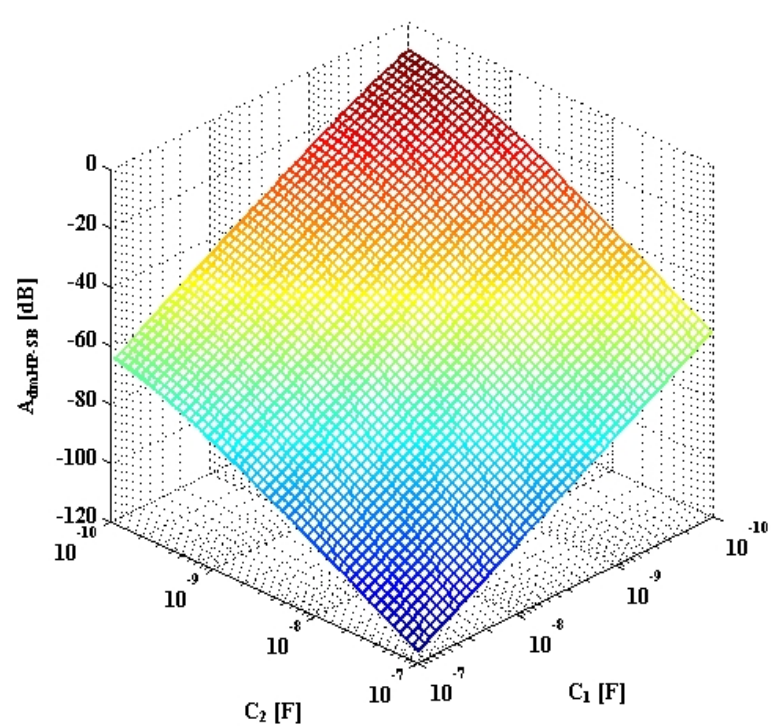

Fig. 6. Variant for attainment of maximal attenuation for $Q=0.707$.

\section{B. Simulations and Experimental Measurements of Transfer Functions}

According to the above given optimization graph Fig. 5, in order to obtain the minimal shift in pole-frequency the optimal values of capacitors should be within the range of $C_{1}=C_{2}=<1,10>\mathrm{nF}$. From Fig. 6 , to achieve at least $40 \mathrm{~dB}$ or higher attenuation in the stop-band of the high-pass filter, the values of capacitors should be selected $C_{1}=C_{2}=$ $1 \mathrm{nF}$ or higher. Therefore, for simulations and experimental measurements, the values of capacitors $C_{1}=C_{2}=1 \mathrm{nF}$ were selected and the values of resistors were determined as $R_{1}=R_{3}=1125 \Omega$ and $R_{2}=2251 \Omega$ to obtain the polefrequency $100 \mathrm{kHz}$ and quality factor $Q=0.707$. During the simulations and experimental measurements, the resistor values were selected from the E24 series, i.e. $R_{1}=R_{3}=1100 \Omega$ and $\mathrm{R}_{2}=2200 \Omega$. These passive element values were used for all types of measurements. As the filter uses one DDCC and two DVCCs, for simulations and the experimental measurements three universal current conveyors UCC-N1B 0520 had to be used, [35].

The performance of the filter has firstly been verified by simulations and furthermore by experimental measurements, whereas the obtained frequency responses of the universal pseudo-differential second-order filter working in voltage mode is shown in Fig. 7.

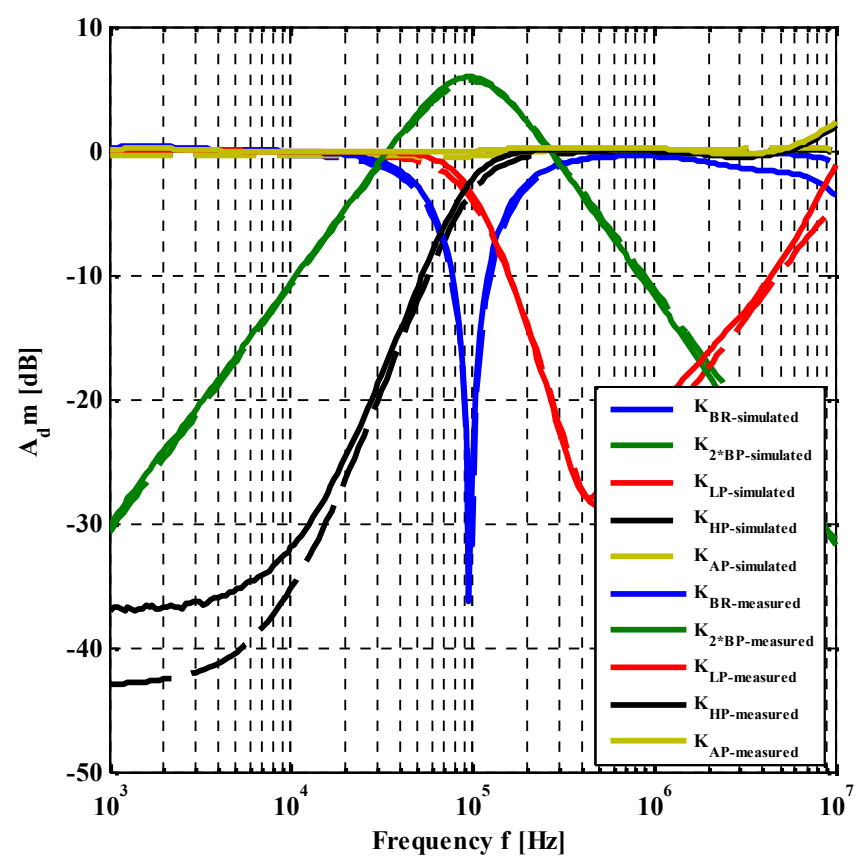

Fig. 7. Simulated (dashed line) and measured (solid line) magnitude responses of the pseudo-differential filter responses BR, BP, LP, HP, AP.

From both the simulations and experimental measurements shown in Fig. 7 we can claim that they are in very good agreement with the theory and performance analysis described in Section IV. B-2. Lower attenuation of the high-pass response in the stop-band than expected during the optimization phases is caused by the parasitic characteristics of the active elements, especially lower impedance of the output terminals $Z$. Increase of the magnitude of the low-pass filter at the frequency of approximately $450 \mathrm{kHz}$ is caused by the non-ideal behavior of the active elements. Assuming parasitic properties, a 
detailed analysis of the transfer function shows non-zero term with the Laplace operator $s^{2}$, causing increase of the magnitude and could be already observed during the simulations. The other frequency responses agree to theory and the performed optimization phases.

In Fig. 8, the measured common-mode rejection ratios of all types of the corresponding transfer functions are shown. The reached value of CMRR is approximately $36 \mathrm{~dB}$ and is constant up to $1 \mathrm{MHz}$. The drop of CMRR above this frequency is caused by the real behavior of the UCC-N1B 0520 realizing the $\mathrm{DDCC}_{1}$ from Fig. 3 and different polefrequency of the voltage-gains $\delta_{1}$ and $\delta_{2}$ [35]. Next to CMRR the THD of the low-pass filter response has been measured. Evaluating THD, the first major harmonic components were considered, whereas the $1 \mathrm{kHz}$ input signal has been applied to $V_{\text {in }}$.

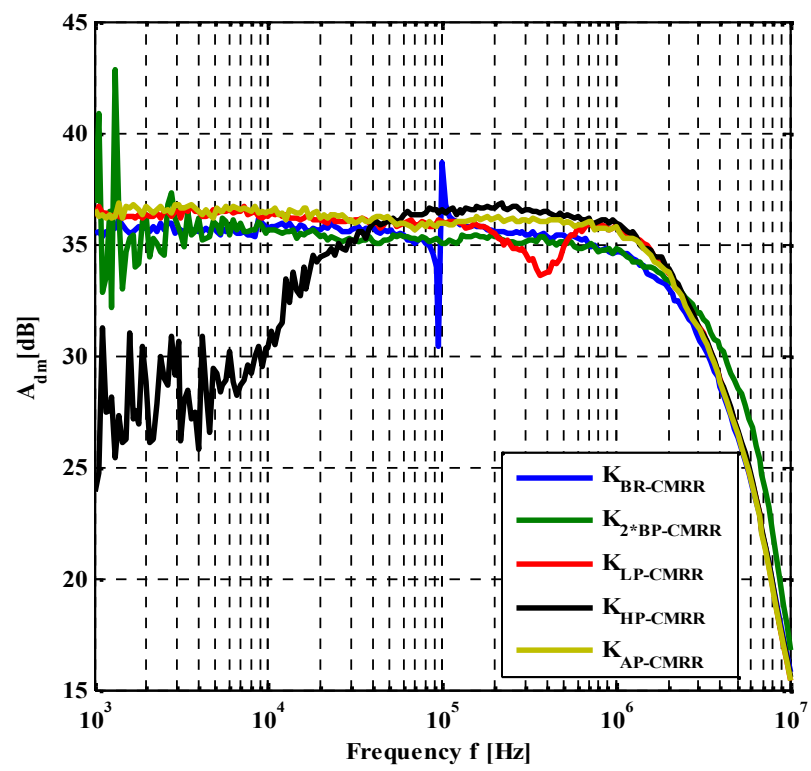

Fig. 8. The common-mode rejection ratio (CMRR) obtained by experimental measurements.

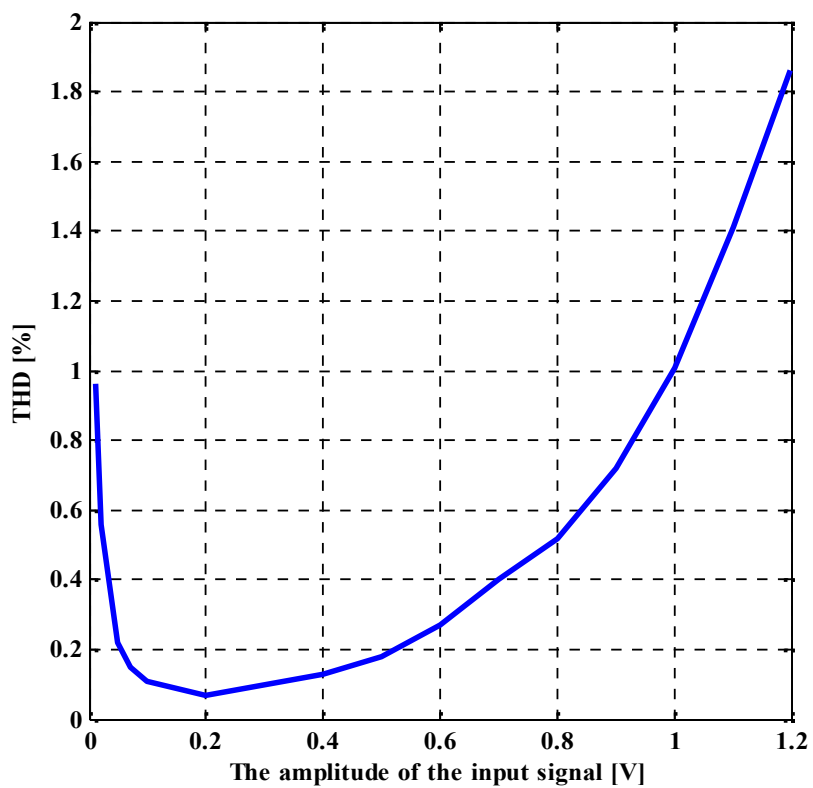

Fig. 9. The measured THD for the LP response of the proposed pseudodifferential filter.

From Fig. 9 it can be observed that the total harmonic distortion is below $1 \%$ for the amplitude of the input signal up to $1 \mathrm{~V}$. Increase of THD for higher amplitudes of the input signal is caused by the saturation of the active elements, i.e. UCC-N1B 0520, being supplied with the $\pm 1.65 \mathrm{~V}$ supply voltages.

To measure the performance of the pseudo-differential filter in practice the network analyser 4395A has been used. Therefore, additional single-to-differential (S/D) and differential-to-single $(\mathrm{D} / \mathrm{S})$ voltage converters, as shown in Fig. 10, have been used. The S/D convertor uses the readily available $\mathrm{AD} 8476$ [36], whereas the $\mathrm{D} / \mathrm{S}$ converter is composed of AD 8429 [37]. To enable the evaluation of the

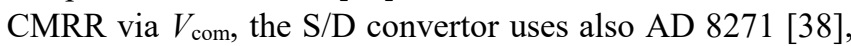
which can be found in more detail in [31].

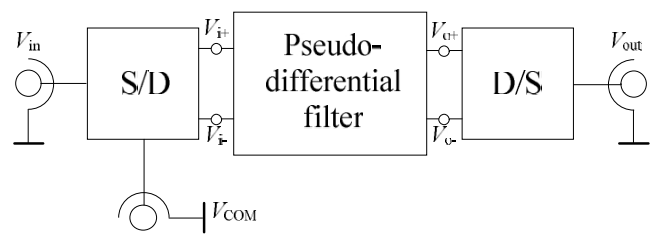

Fig. 10. Block diagram for the practical part.

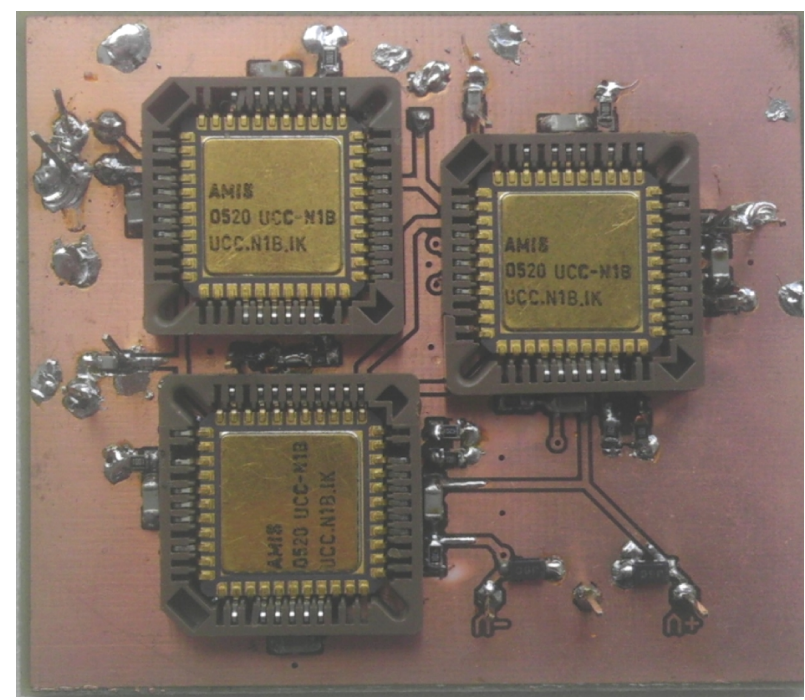

Fig. 11. Prototype PCB of the pseudo-differential filter.

\section{CONCLUSIONS}

In this paper we presented a new current conveyor-based universal pseudo-differential filter working in voltage mode. The proposed filter employs one differential difference current conveyor and two differential voltage current conveyors as active elements, and five passive elements (two capacitors and three resistors), whereas all are grounded. The proposed structure is able to realize all five standard frequency filter responses. The circuit has a high-impedance input and is sufficiently suppressing the common-mode signal. Assuming the parasitic parameters of the active elements, the values of the passive elements have been optimized in terms of minimal shift of the pole-frequency and to obtain the maximum stop-band attenuation of the high-pass filter response. The functionality and performance of the filter has been verified by both simulations and experimental measurements. The total harmonic distortion is less than $1 \%$ for the amplitude of the input signal below 1 $\mathrm{V}$. The value of CMRR reached by measurements is $36 \mathrm{~dB}$ and can be generally increased by proper selection of the active elements. Based on these measurements we can say 
that the filter offers sufficient rejection of common-mode signal in respect to its complexity.

\section{REFERENCES}

[1] T. M. Abuelma'atti, A. Bentrcia, "A novel mixed-mode OTA-C universal filter", International Journal of Electronics, vol. 92, no. 7, 2005, pp. 375-383. [Online]. Available: http://dx.doi.org/10.1080/ 08827510412331295009

[2] Ch. Lee, "Multiple-mode OTA-C universal biquad filters", Circuits, Systems, and Signal Processing, vol. 29, no. 2, pp. 263-274, 2010. [Online]. Available: http://dx.doi.org/10.1007/s00034-009-9145-0

[3] Ch. Chun-Ming, M. Bashir, A. Hashimi, S. Yichuang, N. J. Ross, "New high-order filter structures using only single-ended-input OTAs and grounded capacitors", IEEE Trans. Circuits and Systems-II, vol. 51, no. 9, pp. 458-463, 2004. [Online]. Available: http://dx.doi.org/10.1109/TCSII.2004.831426

[4] S. Hintea, P. Farago, M. N. Roman, G. Oltean, L. Festila, "A Programmable Gain Amplifier for Automated Gain Control in Auditory Prostheses", J. Med. Biol. Eng., vol. 31, no 3, 2011, pp. 185-192. [Online]. Available: http://dx.doi.org/ 10.5405/jmbe.740

[5] W. Tangsritat, "Single-input three-output electronically tunable universal current-mode filter using current follower transconductance amplifiers", AEU - International Journal of Electronics and Communications, vol. 65, no. 10, pp. 783-787, 2011. [Online]. Available: http://dx.doi.org/10.1016/j.aeue.2011.01.002

[6] W. Tangsritat, "Novel current-mode and voltage mode universal biquad filters using single CFTA", Indian Journal of Engineering and Materials Sciences, vol. 17, pp. 99-104, 2010.

[7] W. Tangsritat, W. Surakampontorn, "Design of electronically tunable ladder filters using current-controlled current differential buffered amplifiers", in Proc. 2004 IEEE Region 10th Conf. (TENCON 2004), Chiang Mai, Thailand, 2004, pp. 368-371. [Online]. Available: http://dx.doi.org/10.1109/TENCON.2004.1414946

[8] F. Kacar, S. Menekay, "Realization of a CMOS current differencing buffer amplifier and its filter application", in Proc. 7th Int. Conf. Electrical and Electronics Engineering (ELECO 2011), Bursa, Turkey, 2011, pp. 337-340.

[9] H. Chen, "Current-mode dual-output ICCII-based tunable universal biquadratic filter with low-input and high output impedances", International Journal of Circuit Theory and Applications, vol. 42, no. 4, pp. 376-393, 2012. [Online]. Available: http://dx.doi.org/10.1002/cta.1858

[10] M. A. Soliman, "Current mode filters using two output inverting CCII", International Journal of Circuit Theory and Applications, vol. 36, no. 7, pp. 875-881, 2008. [Online]. Available: http://dx.doi.org/10.1002/cta.463

[11] E. Yuce, A. Kircay, S. Tokat, "Universal resistorless current-mode filters employing CCCIIs", International Journal of Circuit Theory and Applications, vol. 36, no. 5-6, pp. 739-755, 2008. [Online]. Available: http://dx.doi.org/10.1002/cta.455

[12] F. Kacar, A. Yesil, "FDCCII-based electronically tunable voltagemode biquad filter", International Journal of Circuit Theory and Applications, vol. 40, no. 4, pp. 377-383, 2012. [Online]. Available: http://dx.doi.org/10.1002/cta.731

[13] F. Kacar, B. Metin, H. Kuntman, O. Cicekoglu, "Current-mode multifunction filters using a single FDCCII", in Proc. Int. Conf. Electricaland Electronics Engineering (ELECO 2009), Bursa, Turkey, 2009, pp. II-54-II-57. [Online]. Available: http://dx.doi.org/10.1109/ELECO.2009.5355234

[14] M. A. Soliman, "Generation and classification of Kerwin-HuelsmanNewcomb circuits using the DVCC", International Journal of Circuit Theory and Applications, vol. 37, no. 7, pp. 835-855, 2009. [Online]. Available: http://dx.doi.org/10.1002/cta.503

[15] H. Chen, "Tunable versatile current-mode universal filter based on plus-type DVCCs", AEU - International Journal of Electronics and Communications, vol. 66, no. 4, pp. 332-339, 2012. [Online]. Available: http://dx.doi.org/10.1016/j.aeue.2011.08.010

[16] J. W. Horng, W. Y. Chiu, "High input impedance DDCC-based voltage-mode universal biquadratic filter with three inputs and five outputs", Indian Journal of Engineering and Materials Sciences, vol. 18, pp. 183-190, 2011

[17] J. W. Horng, Ch-M. Wu, N. Herencsar, "Fully differential first-order allpass filters using a DDCC", Indian Journal of Engineering and Materials Science, vol. 21, pp. 345-350, 2014.

[18] P. B. Basyurt, E. Bonizzoni, D. Y. Aksin, F. Maloberti, "Voltage reference architectures for low-supply-voltage low-power applications", Microelectronics Journal, vol. 46, no. 11, pp. 1012 1019, 2015. [Online]. Available: http://dx.doi.org/10.1016/j.mejo .2015 .08 .005

[19] A. H. M. Shirazi, H. Rasthian, R. Molavi, T. Taris, H. M. Lavasani, S. Mirabbasi, "On the design of combined LNA-VCO-mixer for lowpower and low-voltage CMOS receiver front-ends", Microelectronics Journal, vol. 57, pp. 34-47, 2016. [Online]. Available: http://dx.doi.org/10.1016/j.mejo.2016.07.013

[20] M. A. Ibrahim, S. Minaei, H. Kuntman, "A $22.5 \mathrm{MHz}$ current-mode KHN-biquad using differential voltage current conveyor and grounded passive elements", AEU - Int. J. Electron. Commun., vol. 59, no. 5, pp. 311-318, 2005. [Online]. Available: http://dx.doi.org/10.1016/j.aeue.2004.11.027

[21] C. F. T. Soares, G. S. de Moraes, A. Petraglia, "A lowtransconductance OTA with improved linearity suitable for lowfrequency Gm-C filters”, Microelectronics Journal, vol. 45, no. 11, pp. 1499-1507, 2014. [Online]. Available: http://dx.doi.org/10. 1016/j.mejo.2014.07.008

[22] N. Herencsar, J. Jerabek, J. Koton, K. Vrba, S. Minaei, I. C. Goknar, "Pole frequency and pass-band gain tunable novel fully-differential current-mode all-pass filter", in Proc. IEEE Int. Symposium on Circuits and Systems (ISCAS 2015), Lisbon, Portugal, 2015, pp. 2668-2671. [Online]. Available: http://dx.doi.org/10.1109 /ISCAS.2015.7169235

[23] J. Koton, N. Herencsar, K. Vrba, J. Jerabek, "Digitally adjustable current amplifier and its application in fully differential current-mode band-pass filter design", Elektrorevue Internet Journal, vol. 1, pp. 47-52, 2010.

[24] M. A. Ibrahim, H. Kuntman, "A novel high CMRR high input impedance differential voltage-mode KHN-biquad employing DODDCCs", AEU - International Journal of Electronics and Communications, vol. 58, no. 6, pp. 429-433, 2004. [Online]. Available: http://dx.doi.org/10.1078/1434-8411-54100266

[25] L. Langhammer, J. Jerabek, J. Polak, "Tunable fully-differential filters designed using signal-flow graphs method", Elektrorevue Internet Journal, vol. 6, no. 3, pp. 38-48, 2015.

[26] J. Jerabek, R. Sotner, K. Vrba, I. Koudar, "A fully differential universal tunable filter with current active elements", Elektrorevue Internet Journal, vol. 2010, no. 7, pp. 1-6, 2010.

[27] K. Chen, "Design of differential filters for high-speed signal chains", Texas Instruments, SLWA053B, rev. April 2010.

[28] J. Jerabek, J. Koton, R. Sotner, K. Vrba, “Adjustable band-pass filter with current active elements: two fully-differential and single-ended solutions", Analog Integrated Circuits and Signal Processing, vol. 74, no. 1, pp. 129-139, 2013. [Online]. Available: http://dx.doi.org/10.1007/s10470-012-9942-4

[29] U. Tietze, Ch. Schenk, E. Gamm, Electronic circuits: handbook for design and application. Berlin: Springer. 2008, p. 1543. [Online]. Available: http://dx.doi.org/10.1007/978-3-540-78655-9

[30] T. Kuehl. Using the infinite-gain, MFB filter topology in fully differential active filters. Texas Instruments Incorporated, Analog Applications Journal, 2009. [Online]. Available: www.ti.com/aaj

[31] J. Koton, N. Herencsar, O. Sladok, J. Horng, "Pseudo-differential second order band reject filter using current conveyors", $A E U$ International Journal of Electronics and Communications, vol. 70, no. 6, pp. 814-821, 2016. [Online]. Available: http://dx.doi.org/ 10.1016/j.aeue.2016.03.009

[32] J. Koton, N. Herencsar, J.-W. Horng, "Differential second-order voltage-mode all-pass filter using current conveyors", Elektronika ir Elektrotechnika, vol. 22, no. 5, pp. 52-57, 2016. [Online]. Available: http://dx.doi.org/10.5755/j01.eie.22.5.16344

[33] R. Raut, Swamy MNS. Modern Analog Filter Analysis and Design: A Practical Approach. Weinheim: Wiley-VCH; 2010, p. 355.

[34] S. Maheshwari, A. Gangwar, "Versatile voltage-mode universal filter using differential difference current conveyor", Circuits and Systems, vol. 2, no. 3, pp. 210-216, 2011. [Online]. Available: http://dx.doi.org/10.4236/cs.2011.23030

[35] Datasheet UCC-N1B 0520. Universal current conveyor (UCC) and second-generation current conveyor $(\mathrm{CCII}+/-)$, rev. 1. Brno University of Technology, On Semiconductor Ltd.; 2012.

[36] Datasheet AD 8476. Low power, unity gain, fully differential amplifier and ADC driver, Analog Devices, rev. B.; 2012.

[37] Datasheet $\mathrm{AD} 8429.1 \mathrm{nV} / \sqrt{ } \mathrm{Hz}$ low noise instrumentation amplifier, Analog Devices, rev. 0; 2011.

[38] Datasheet AD 8271. Programmable gain precision difference amplifier, Analog Devices, rev. 0; 2009. 